\title{
An Approximation Model of AC Resistance for Inductor and Transformer Windings with Partial Layers
}

\author{
Zhan Shen ${ }^{*, * *}$ Non-member, Zhiguang Li* Non-member \\ Long Jin* Non-member, Huai Wang** Non-member
}

(Manuscript received July 18, 2019, revised March 21, 2020)

\begin{abstract}
This paper proposes an approximation model of the AC resistance for windings with partial layers. It introduces the concept of the number of layers for the partial winding without theoretical support. It is simpler compared to the original partial layer equation and and has a good accuracy. The error between the approximation and the original equation is derived and compared analytically. The approximation method is verified in two case studies with simulation results of the finite element method and experimental results. Based on the approximation equation, an optimization method is proposed for the winding with partial layers. This can provide a minimum $\mathrm{AC}$ resistance within the design restrictions and can be applied to inductors and transformers. Finally, the optimization method is verified in a case study of a $20 \mathrm{kHz}$ transformer winding design.
\end{abstract}

Keywords: winding resistance, transformers, inductors, partial layer windings, winding optimization

\section{Introduction}

With the promotion of wide-band-gap (WBG) devices, the operating frequency and power density of the power electronic converters increase continuously. It brings a significant challenge to the loss modeling and optimization of passive devices. Normally the magnetics contribute considerable loss to the converter, including the magnetic core loss and the winding resistive loss ${ }^{(1)-(4)}$. Therefore the modeling of the winding resistance is essential, especially in high frequency, power, and power density applications with complicated winding configurations.

There are two kinds of windings: full layer and partial layer, as illustrated in Fig. 1. The full layer takes all of the window height space in $z$-direction. The partial layer only appears in the last layer of the winding, and is with the fewer number of turns than the full layer.

There is mature research on the AC resistance modeling of windings with full layers. Dowell proposed the analytical equation for the transformer winding resistance in the Cartesian coordinate system ${ }^{(5)}$. It is improved in (6) by considering the two-dimensional magnetic field distribution and porosity factor. Another method is to build the model in the cylindrical coordinate system and is named Ferreira's equation ${ }^{(7)(8)}$. It is originally for round wires, and is extended to other conductor shapes with the equivalent copper area principle ${ }^{(9)}$. These methods are compared and summarized in (10). The analytical models for the Litz wire are proposed in (11)-(13). Recent advances use the results of the finite element method (FEM) to fit the equation for the AC resistance ${ }^{(14)(15)}$.

\footnotetext{
${ }^{*}$ Southeast University

Sipailou 2, Nanjing 210096, China

** Aalborg University

Pontoppidanstraede 101, Aalborg 9220, Denmark
}

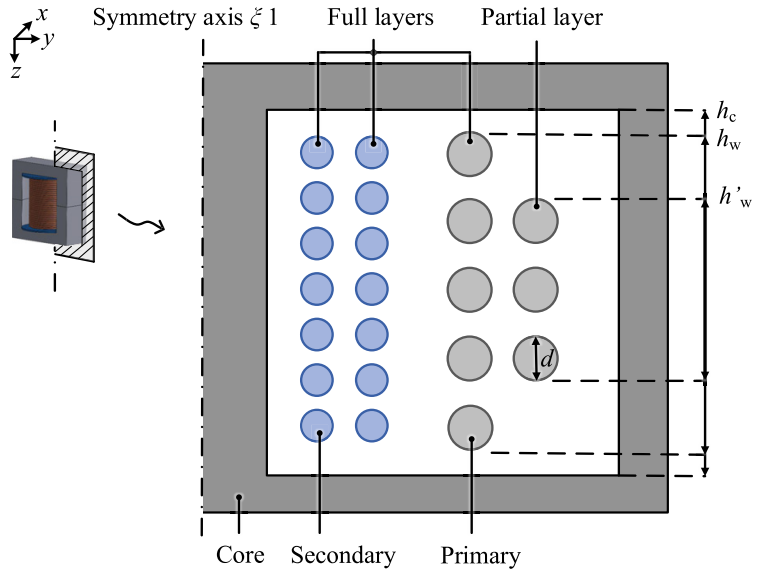

Fig. 1. Transformer with full and partial layer windings

For the partial layer, the authors proposed an original partial layer equation in (16). However, its complexity restricts the application. In this paper, an alternative method is proposed by using the approximation of Dowell's equation and the concept of the number of layers for the partial winding. It is simple and without theoretical background. The accuracy and difference compared with the original partial layer equation are discussed in detail.

For the winding optimization, there are computer-aid and analytical methods, respectively. The first one sweeps all the possible cases within restrictions and requires large computational resources and time ${ }^{(3)}$. For the analytical method, Hurley et al. proposed a simple equation to calculate the optimal wire diameter for the minimum winding resistance ${ }^{(17)}$. It optimizes the winding in different current waveforms and requires the predefined number of layers $m$ as the design input. However, $m$ usually is unknown before the winding 
design. The authors proposed another method based on Hurley's equation ${ }^{(16)(18)}$. It only requires the number of total turns $N$ and core window dimensions as design input, which is more practical. In this paper, it is further improved and simplified with the approximation equation.

This paper studies the winding resistance of the partial layer with three major contributions:

1) Propose the approximation equation for the AC resistance of the partial layer. The difference between the original partial layer and approximation equation is derived analytically, and the maximum error case is obtained.

2) Verify the approximation equation with FEM simulation and experimental results in two case studies.

3) Propose a winding resistance optimization method with the approximation equation. Perform a case study for a $20 \mathrm{kHz}$ transformer to verify the winding design method.

\section{AC Windings Resistance of Partial Layer}

In this section, Dowell's equation and the original partial layer equation are reviewed briefly first. Then the approximation model for the partial layers is proposed. It is analyzed and compared with the original partial layer equation in detail. Finally, the approximation equation is verified by the finite element method (FEM) and experimental results.

2.1 Dowell's Equation In 1966, Dowell firstly proposed the closed-form equation for the winding resistance ${ }^{(5)}$

$$
R_{\text {Dowell }}=R_{\mathrm{dc}} F_{\text {Dowell }} \ldots \ldots \ldots \ldots \ldots \ldots \ldots \ldots
$$

with

$$
\begin{aligned}
& F_{\text {Dowell }}=\Delta\left[v_{3}+\frac{2}{3}\left(m^{2}-1\right) v_{2}\right] \\
& \Delta=\frac{d_{\mathrm{w}}}{\delta} \\
& v_{2}=\frac{\sinh \Delta-\sin \Delta}{\cosh \triangle+\cos \triangle}, \quad v_{3}=\frac{\sinh (2 \triangle)+\sin (2 \triangle)}{\cosh (2 \triangle)-\cos (2 \triangle)}
\end{aligned}
$$

where $R_{\text {Dowell }}$ and $R_{\mathrm{dc}}$ are the $\mathrm{AC}$ and DC winding resistance, respectively, $F_{\text {Dowell }}$ is the resistance factor, $\Delta$ is the penetration ratio, $d_{\mathrm{w}}$ is the thickness of the foil, $\delta$ is the skin depth, $m$ is the number of winding layers, $v_{3}$ and $v_{2}$ are the coefficients for the skin and proximity effects, respectively. For round wires with diameter $d$, it is transferred to the equivalent thickness of the foil with the equivalent area transformation

$$
d_{\mathrm{w}}=\sqrt{\frac{\pi}{4}} d .
$$

2.2 Original Partial Layer Equation The original partial layer equation for the winding with $m$ full layers and one partial layer as $m+1$ layer is ${ }^{(16)}$

$$
R_{\text {ori. }}=R_{\mathrm{dc}} F_{\text {ori. }}
$$

with

$$
F_{\text {ori. }}=\Delta v_{3}+\frac{4 m^{3}-4 m-3 k+3 k(2 m+k)^{2}}{6(m+k)} \Delta v_{2}
$$

The partial coefficient $k$ is defined as

$$
k=\frac{t_{0}}{t}=\frac{h^{\prime}{ }_{\mathrm{w}}}{h_{\mathrm{w}}}
$$

where $t, h_{\mathrm{w}}, t_{0}$, and $h_{\mathrm{w}}^{\prime}$ are the number of turns and winding height in full and partial layers, respectively. When $k=0$ or $k=1$, (6) transfers to Dowell's equation (1) with $m$ or $m+1$ layers.

\subsection{Proposed Approximation of Partial Layer Equa-}

tion Equation (6) considers the difference of the field intensity between the full layer and partial layers theoretically. However, it is complex and not convenient for applications. An alternative solution is to use Dowell's equation to approximate equation (6). The new-defined the number of layers for the partial winding $m_{p}$ is

$$
m_{p}=m+k \cdots
$$

The original number of layers $m$ is an integer and $m_{p}$ is a fraction or integer.

Substituting $m_{p}$ into (1) is the approximations model of the winding with partial layers

$$
R_{\text {approx. }}=R_{\mathrm{dc}} F_{\text {approx. }}
$$

with

$$
F_{\text {approx. }}=\Delta\left[v_{3}+\frac{2}{3}\left(m_{p}^{2}-1\right) v_{2}\right]
$$

2.4 Difference between Dowell, Original Partial Layer, and Approximation Equations A case study with a designed inductor is presented to compare the difference between the three equations. It is with PQ 50/50 core shape, 3C91 core material with the approximate relative permeability of $3000,1.56 \mathrm{~mm}$ round wire, number of full layers $m=5$, turns in full layers $t=10$, and sweeping turns of partial layer $t_{0}$ from 0 to 10 . When $t_{0}=10$, the inductor turns to $m=6$ full winding scenario.

The comparison and the error are in Fig. 2 and Fig. 3, respectively. Dowell's equation leads to significant errors when only considering the full layers. However, with the concept of the number of layers for the partial winding $m_{p}$, the accuracy of the approximation equation improved significantly. The error between the original partial layer and approximation equation is non-linear. The largest error is when the partial coefficient $k$ close to 0.5 , and the error percentage is below $0.1 \%$.

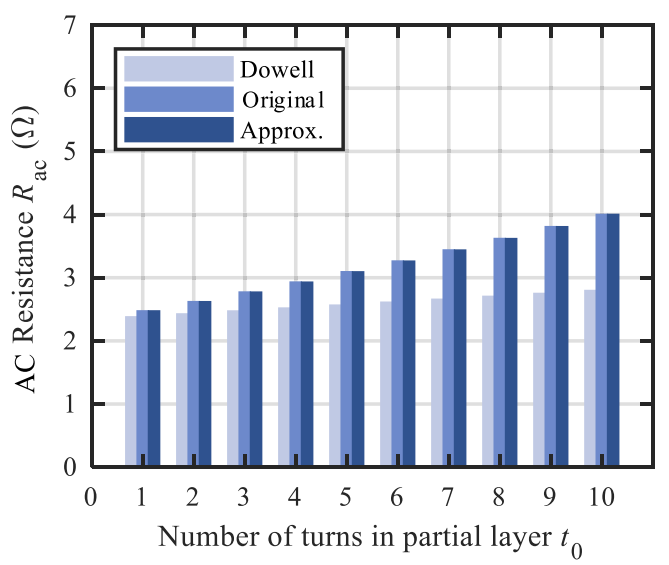

Fig. 2. AC resistance of a designed inductor calculated with Dowell, original partial layer, and approximation equations 


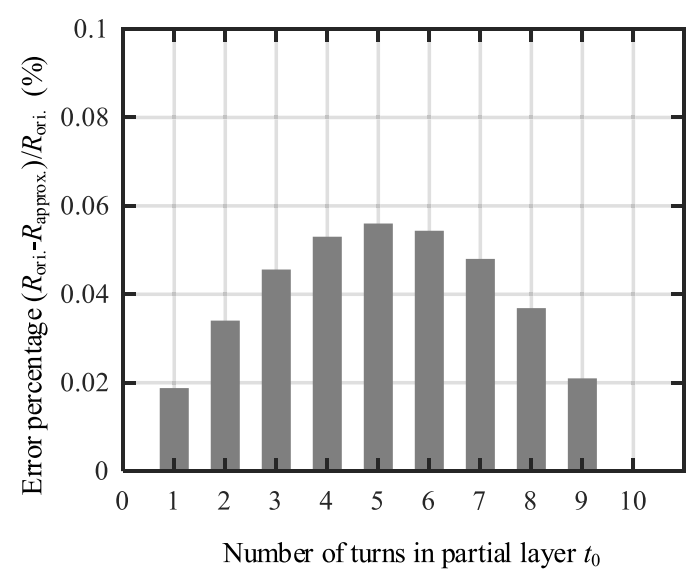

Fig. 3. Error percentage of the approximation equation compared to the original partial layer equation

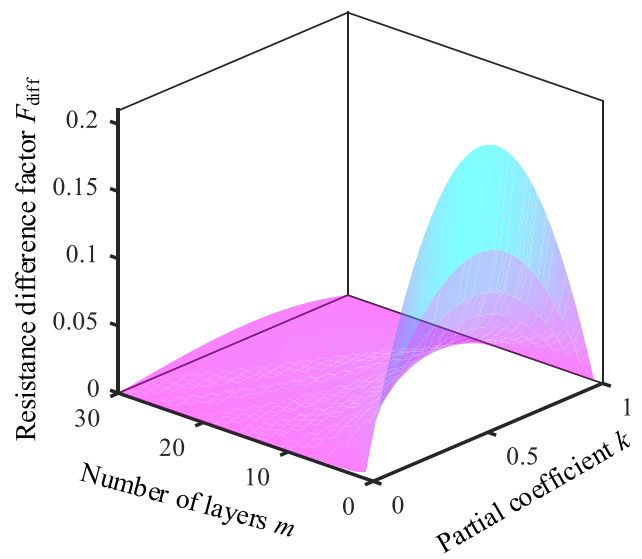

Fig. 4. Resistance factor in terms of different number of layers $m$ and partial coefficient $k$. The maximum resistance factor $\left(F_{\text {diff }}\right) \max =0.21$ is with $k=0.5$ and $m=1$

2.5 Analytical Difference Analysis To compare the difference between the approximation and original partial layer equations, the resistance difference $R_{\text {diff }}$ is defined

$$
\begin{aligned}
R_{\text {diff }} & =R_{\text {ori. }}-R_{\text {approx. }}=R_{\text {dc }}\left(F_{\text {ori. }}-F_{\text {approx. }}\right)=R_{\mathrm{dc}} F_{\text {diff }} \\
& =R_{\text {dc }} \frac{\Delta v_{2}\left(k-k^{3}\right)}{6(m+k)} \ldots \ldots \ldots \ldots \ldots \ldots \ldots \ldots \ldots \ldots \ldots \ldots \ldots \ldots
\end{aligned}
$$

where $F_{\text {diff }}$ is the resistance difference factor. $F_{\text {diff }}$ under different $k$ and $m$ are given in Fig. 4.

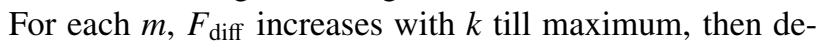
creases. Setting the differential of $F_{\text {diff }}$ with $k$ to zero

$$
\frac{\partial F_{\mathrm{diff}}}{\partial k}=\frac{m\left(1-3 k^{2}\right)-2 k^{3}}{(m+k)^{2}}=0
$$

The $k$ which leads to the maximum $F_{\text {diff }}$ is obtained

$$
k_{\text {max }}(m)=\frac{1}{2}\left(\frac{m^{2}}{q}+q-m\right) .
$$

with

$$
q=\sqrt[\frac{1}{3}]{-m^{3}+2 \sqrt{m^{2}-m^{4}}}
$$

Sweeping $m$ from 1 to $5000, k_{\max }(m)$ is between 0.5 and 0.58 . Therefore, the maximum resistance difference factor

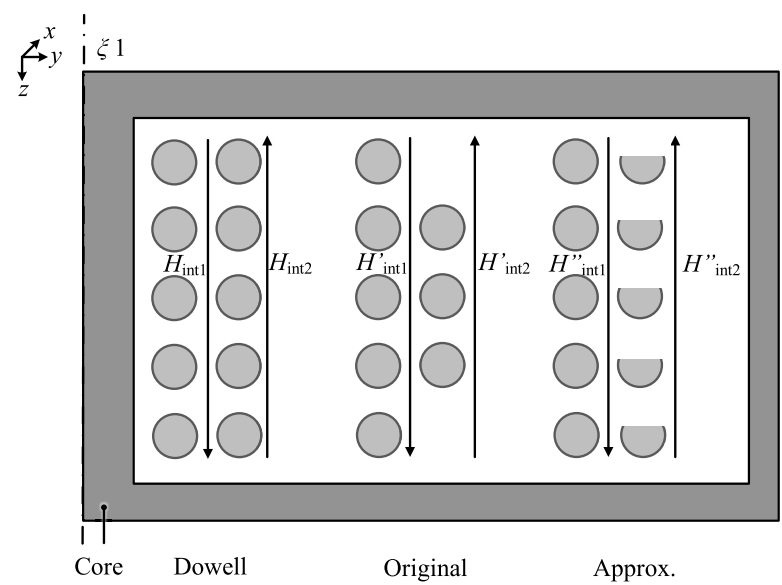

Fig. 5. Difference of the skin magnetic field intensity to calculate the AC resistance factor $F$ with Dowell, original partial layer, and approximation equation

$\left(F_{\text {diff }}\right)_{\max }$ happens when $k$ is between 0.5 and 0.6. With the approximation equation, the highest error happens approximately when it is the half layer. The maximum AC resistance error $R_{\mathrm{diff}}=0.21 R_{\mathrm{dc}}$ when $m=1$, and decreases with the increase of $m$.

Figure 5 illustrates the difference of each equation in terms of their assumptions of the magnetic field intensity. For the approximation equation, the assumptions for the original Dowell's equation is still valid:

- The permeability of the core is much larger than the air, and the magnetic field strength in the core $H \approx 0$.

- The current flows only in the $x$-direction, therefore the $H$ field is in the $y z$-plane.

- The wires occupy the window in height directions, $h_{\mathrm{w}} \approx h_{\mathrm{c}}$, therefore the $H$ field in the window is onedimensional in $z$-direction.

For original partial layer equation, the skin and proximity magnetic field intensity of the partial layer is

$$
\begin{aligned}
& H_{\text {int } 1}^{\prime}=-H_{\text {int } 2}^{\prime}=\frac{k \hat{I}}{2 h_{\mathrm{c}}} \ldots \ldots \\
& H_{\text {ext } 1}^{\prime}=H_{\text {ext } 2}^{\prime}=\frac{2 m \hat{I}+k \hat{I}}{2 h_{\mathrm{c}}} .
\end{aligned}
$$

The total current in the partial layer decreases to $k \hat{I}$ while the height of the window keeps the same as $h_{\mathrm{c}}$. For Dowell's equation, $k=1$.

When using Dowell's equation to approximate the original partial layer equation, the field intensity path in the core is kept the same to fulfill the assumptions. The skin and proximity magnetic field intensity of the partial layer is

$$
\begin{aligned}
& H^{\prime \prime}{ }_{\text {int } 1}=-H^{\prime \prime}{ }_{\text {int } 2}=\frac{\hat{I}}{2 h_{\mathrm{c}}} \ldots \ldots \ldots \ldots \ldots \ldots \ldots \ldots \ldots \ldots \ldots \ldots \\
& H^{\prime \prime}{ }_{\text {ext } 1}=H^{\prime \prime}{ }_{\text {ext } 2}=\frac{(2 m+1) \hat{I}}{2 h_{\mathrm{c}}} \ldots \ldots \ldots \ldots \ldots \ldots \ldots
\end{aligned}
$$

Therefore the field intensities of the approximation equation are the same. The coefficient to approximate the winding resistance increment is

$$
\frac{R_{\text {approx }}(m+1)-R_{\text {Dowell }}(m)}{R_{\text {Dowell }}(m+1)-R_{\text {Dowell }}(m)}
$$




$$
\begin{aligned}
& =\frac{k \Delta v_{3}+2 / 3 * \Delta v_{2} k\left(3 m k+3 m^{2}+k^{2}-1\right)}{\Delta v_{3}+2 \Delta v_{2} m(1+m)} \\
& \neq k \ldots \ldots \ldots \ldots \ldots \ldots \ldots \ldots \ldots \ldots \ldots \ldots \ldots \ldots \ldots \ldots \ldots \ldots \ldots \ldots \ldots
\end{aligned}
$$

The increase of the AC resistance due to partial layer is nonlinear with $k$. Therefore, the approximation equation is with no theoretical basis, though with good accuracy.

Finally, the decrease of the field intensity due to the partial layer (16) (17) is different from the concept of porosity factor. The porosity factor $\eta$ is defined by the height of the winding to the height of the core

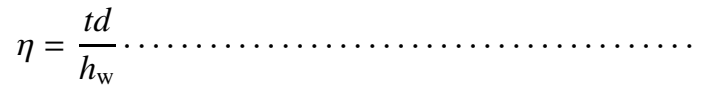

It is to improve Dowell's equation through the penetration ratio $\Delta$

$$
\Delta=\sqrt{\eta} \frac{d_{\mathrm{w}}}{\delta}
$$

$\eta$ is to modify the relation between the winding and the core window, while the partial layer equation focuses on the relation between the partial layer and full layer. Therefore they do not conflict with each other, and $\eta$ is applicable for both the original partial layer and approximation equations.

2.6 Model Verification: Two Case Studies In case 1, two transformers with the same secondary winding, but with and without partial layer as the primary winding is built and tested, named Prototype 1 (P1) and Prototype 2 (P2). The winding configuration is in Table 1. $\mathrm{P} 1$ and $\mathrm{P} 2$ are with the same PQ50/50 core shape, 3C91 core material, and insulation design. The FEM simulations are performed with the software FEMM ${ }^{(19)}$. P2 and its simulation results are in Fig. 6.

The AC resistance of the transformer is measured with the Aglient E5061B network analyzer, as illustrated in Fig. 7. $R_{1}$, $R_{2}, L_{1 \sigma}, L_{2 \sigma}$ are the primary and secondary winding resistance and leakage inductance of the transformer, respectively, $R_{\mathrm{m}}$ and $L_{\mathrm{m}}$ are the main resistance and inductance, respectively. The secondary side is short-circuited, and the AC resistance is measured from the primary side with the network analyzer. $R_{\mathrm{m}}$ is much larger than $R_{1}$ and $R_{2}$. Therefore, $R_{\mathrm{m}}$ is neglected in the circuit. The measured AC resistance is the combination of primary and secondary winding resistance

$$
R_{\mathrm{ac}}=R_{1}+\frac{N_{1}^{2}}{N_{2}^{2}} R_{2}
$$

where $N_{1}$ and $N_{2}$ are the number of turns of primary and secondary, respectively. All measurements are carried out at room temperature of $23^{\circ} \mathrm{C}$.

The comparison of the FEM, experimental, and analytical results of P1 and P2 are in Fig. 8. They agree with each other well in a wide frequency range. The errors come from the $H$ field distortion and the edge effect in the winding tip, the simplification of the non-symmetrical structure in $2 \mathrm{D}$ simulation and analytical model, and the measurement error. The difference between the original partial layer and approximation equation is negligible.

Due to the additional partial layer of $\mathrm{P} 2$, there is a large $\mathrm{AC}$ resistance increment compared with P1. Also, the difference increases with the frequency. Therefore, the resistance of the

Table 1. Winding specification of prototype 1,2 , and 3

\begin{tabular}{lcccc}
\hline \hline & \multicolumn{3}{c}{ Primary } & Secondary \\
& P1 & P2 & P3 & P1\& P2\& P3 \\
\hline No. of full layers $m$ & 2 & 2 & 3 & 2 \\
Turns in full layer $t$ & 16 & 16 & 14 & 16 \\
No. of partial layers & 0 & 1 & 0 & 0 \\
Turns in partial layer $t_{0}$ & 0 & 10 & 0 & 0 \\
No. of total turns $N$ & 32 & 42 & 42 & 32 \\
Winding diameter $d(\mathrm{~mm})$ & 1.56 & 1.56 & 1.56 & 1.56 \\
\hline \hline
\end{tabular}

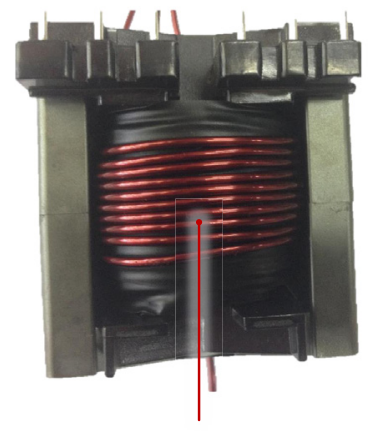

Partial layer of Pri.

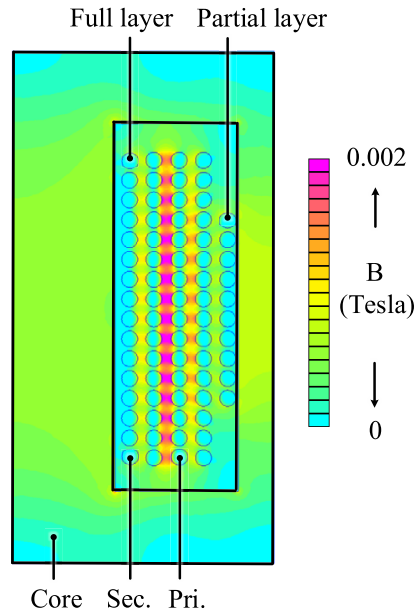

Fig. 6. Prototype $2(\mathrm{P} 2)$ and its FEM simulation model
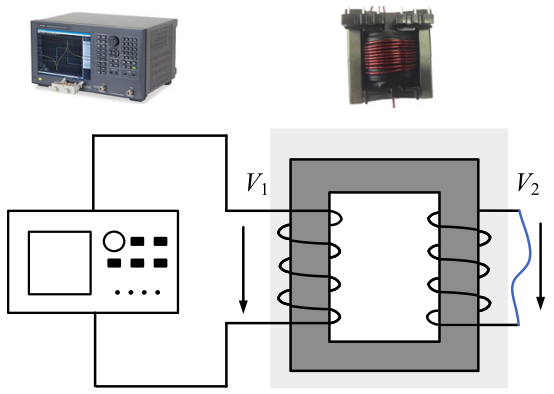

Network Analyzer Primary Secondary: short-circuited

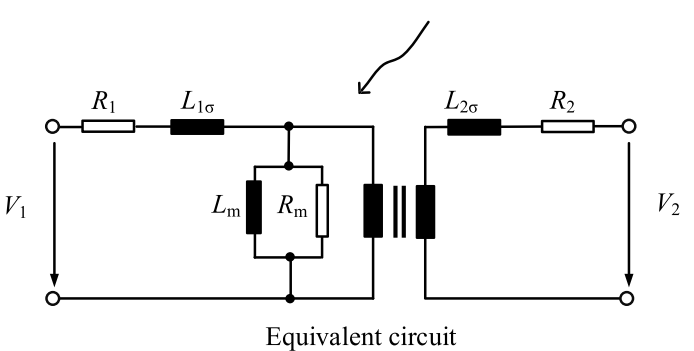

Fig. 7. AC resistance measurement and the equivalent circuit of the transformer

partial layer cannot be neglected in high frequency and large number of layers scenario.

$\mathrm{P} 3$ is designed with the same number of total turns $N$ and turns ratio as P2. Its core is the same as P2. The winding configuration, simulation, and analytical calculation results are shown in Table 1 and Fig. 8, respectively.

P2 is with two full layers and one partial layer. P3 is with three full layers, however, smaller number of turns for each layer. Theoretically, P3 has the same turns $N$ and similar DC resistance as P2. However, the number of layers for P3 and 


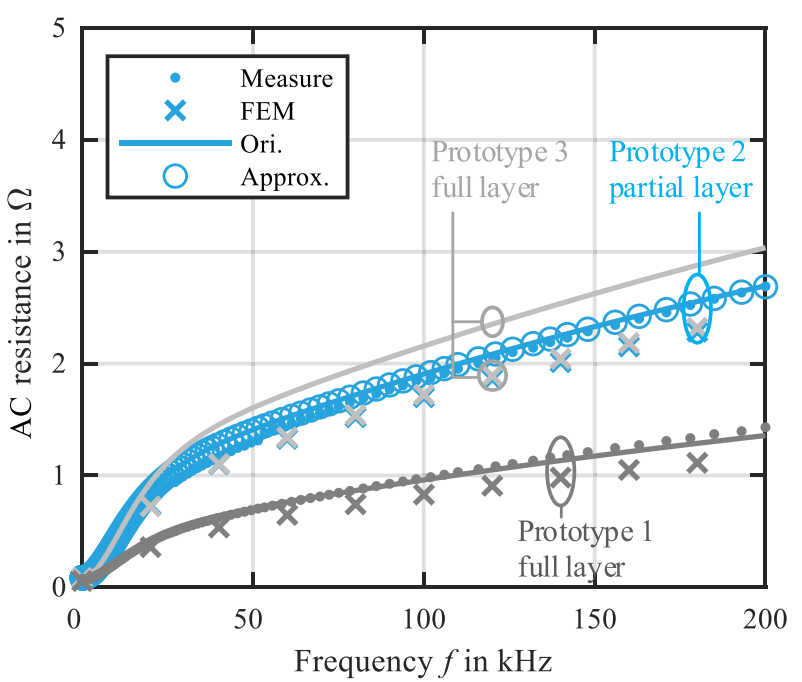

Fig. 8. The analytical, simulation, and experimental results of the ac resistance of prototype $1(\mathrm{P} 1), 2(\mathrm{P} 2)$, and 3 (P3) without, with, and without the partial layer, respectively. The results of $\mathrm{P} 1, \mathrm{P} 2$, and $\mathrm{P} 3$ are with the dark gray, blue, and light gray, respectively

$\mathrm{P} 2$ are 3 and $(2+10 / 16)=2.6$, respectively. The porosity factor $\eta$ for $\mathrm{P} 3$ and $\mathrm{P} 2$ are 0.61 and 0.69 , respectively. Therefore, $\mathrm{P} 3$ has a higher number of layers but a lower porosity factor than $\mathrm{P} 2$. As a result, the $\mathrm{AC}$ resistance factor of $\mathrm{P} 3$ is slightly higher. Finally, the AC resistance of $\mathrm{P} 3$ is also higher.

In general, the comparison of $\mathrm{AC}$ resistance of $\mathrm{P} 2$ and $\mathrm{P} 3$ cannot be applied to other cases. For a specific number of total turns $N$, one solution is to wire windings with higher number of turns per layer and have the partial layer as the last layer, as P2. Another solution is to have lower number of turns per layer but keep all layers the same number of turns, as P3. The first solution achieves smaller AC resistance in some scenarios while in other scenarios does not. To choose between the two solutions requires a generic optimal winding design methodology, as introduced in Section 3.

In case 2 , the fourth transformer named prototype 4 (P4) is built and tested in Fig. 9. The core is the same as P1, and the winding configuration is in Table 2. Compared with P2, the primary winding of $\mathrm{P} 4$ exchanges the position with secondary. So the primary is in the inner position and closer to the symmetry axis. The measurement, analytical, and simulation results of $\mathrm{P} 4$ also shows a good agreement with each other. The AC resistance of the transformer with the same parameters except using 3 full layers for the secondary winding is also designed, as P5. Due to the thin diameter of the secondary wire, there is almost no difference between full layer P5 and partial layer P4 in the low-frequency range. However, with the increase of frequency, the partial layer formula is still necessary.

\section{Optimal Design of Partial Layer winding}

With the approximation equation, the winding design method in the reference (16) is improved with the concept of the number of layers for the partial winding. Compared with the full layer, the partial layer is with more design and configuration flexibility. The AC resistance increases or decreases turn by turn instead of layer by layer. Therefore the design

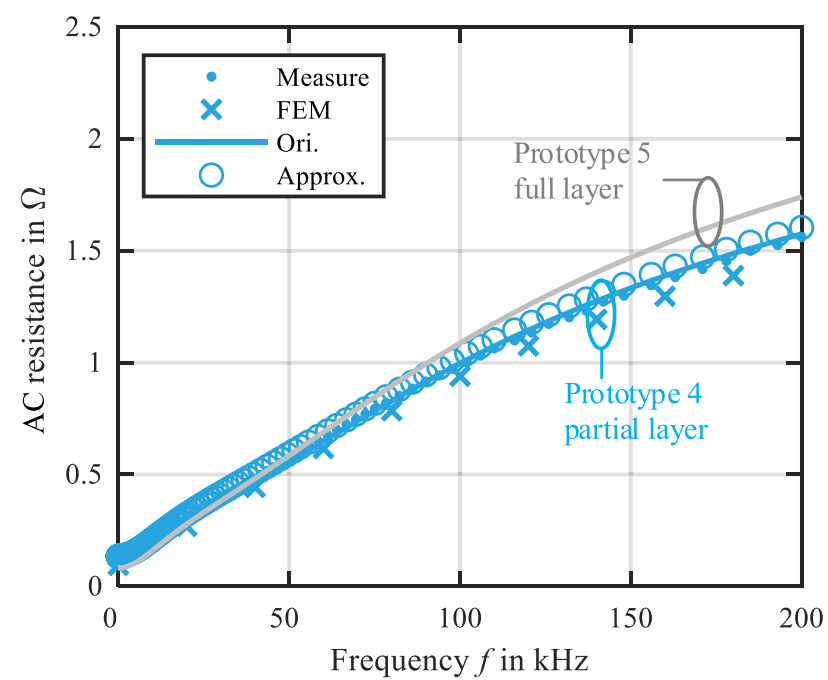

Fig. 9. The analytical, simulation, and experimental results of the AC resistance of P4 and P5 with and without partial layer. The results of P4 and P5 are with the blue and light gray, respectively

Table 2. Winding specification of prototype 4 and 5

\begin{tabular}{lccc}
\hline \hline & Primary & \multicolumn{2}{c}{ Secondary } \\
& P4 \& P5 & P4 & P5 \\
\hline No. of full layers $m$ & 2 & 2 & 3 \\
Turns in full layer $t$ & 16 & 38 & 38 \\
No. of partial layers & 0 & 1 & 0 \\
Turns in partial layer $t_{0}$ & 0 & 20 & 0 \\
No. of total turns $N$ & 32 & 96 & 114 \\
Winding diameter $d(\mathrm{~mm})$ & 1.56 & 0.62 & 0.62 \\
\hline \hline
\end{tabular}

is more precise and controllable. In this section, the conventional methods from references (16) and (17) are shortly reviewed. Then the optimization method is proposed and verified in a case study.

3.1 Optimum Diameter Method In 2013, Hurley et al. proposed an optimization wire diameter formula, which leads to a minimum $\mathrm{AC}$ resistance under the certain $m$. The optimum diameter $d_{\mathrm{opt}}$ is calculated from the optimum penetration ratio $^{(12)(17)}$

$$
d_{\mathrm{opt}}=\Delta_{\mathrm{opt}} \delta=\frac{1}{\sqrt[4]{\Psi}} \sqrt{\tau} \delta \ldots \ldots \ldots \ldots \ldots \ldots \ldots \ldots
$$

with

$$
\Psi=\frac{5 m^{2}-1}{15}, \quad \text { and } \quad \tau=\frac{\omega I_{\mathrm{rms}}}{I^{\prime}{ }_{\mathrm{rms}}} \ldots \ldots \ldots \ldots \ldots
$$

where $I_{\text {rms }}$ and $I^{\prime}{ }_{\text {rms }}$ are the rms and derivation of the current, $\omega=2 \pi f$.

3.2 Winding Design with Original Partial Layer Equation Equation (24) is analytical and convenient for calculation, and $m$ is the precondition. For foil windings, the number of turns $N=m$. Therefore $m$ is known before winding design. However, for round wire windings, $N=m t+t_{0} \neq m$, where $t$ and $t_{0}$ are the turns in full and partial layers, respectively. An alternative method is to sweep $t_{0}$ for different $m^{(16)}$

$$
m=\frac{N-t_{0}}{t} \ldots \ldots \ldots \ldots \ldots \ldots \ldots \ldots \ldots \ldots \ldots
$$




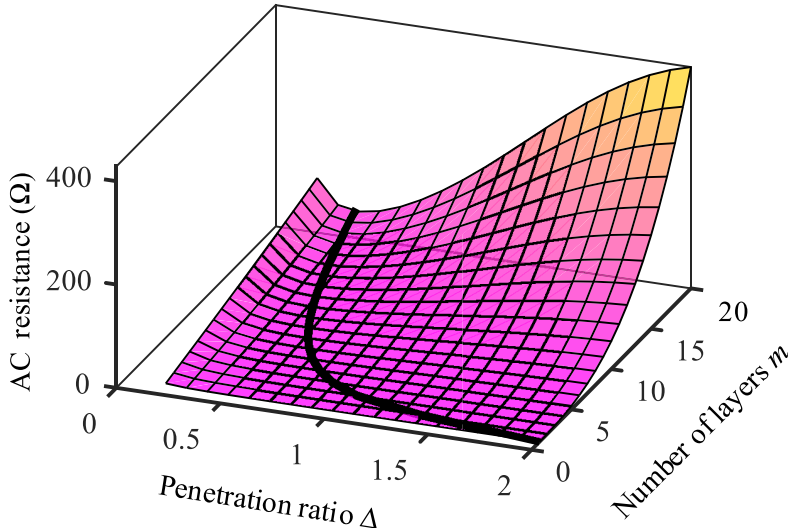

Fig. 10. AC resistance sweeping $\Delta$ and $m$. It is with round conductor and the black line is the optimum design for different $m$

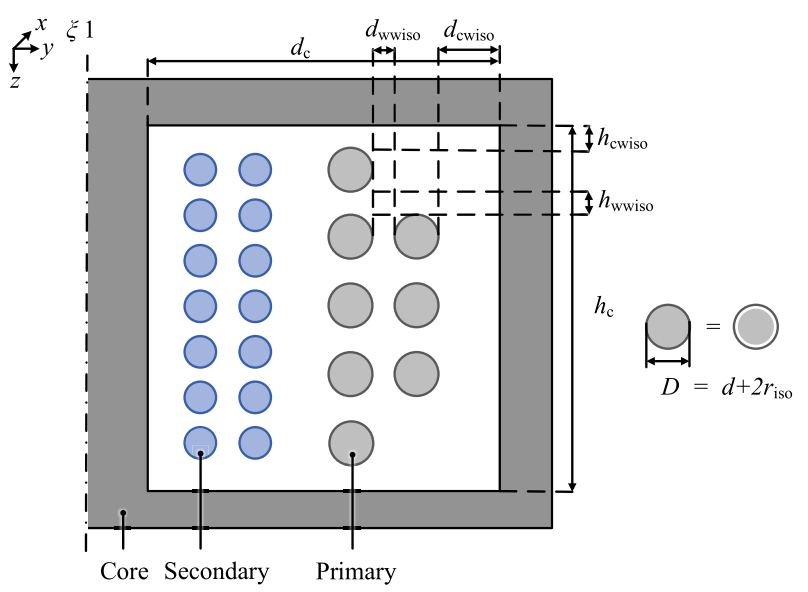

Fig. 11. Winding design parameters and restrictions

Then use the window height $h_{\mathrm{c}}$ and width $d_{\mathrm{c}}$ as restrictions to design the winding in a map

$$
\begin{gathered}
d_{\mathrm{c}} \geq(m+1) d_{\mathrm{opt}}+2(m+1) d_{\text {iso }}+m d_{\text {wwiso }}+2 d_{\text {cwiso }} \\
\ldots \ldots \ldots \ldots \ldots \cdots \cdots \\
h_{\mathrm{c}} \geq t d_{\mathrm{opt}}+2 t d_{\text {iso }}+(t-1) h_{\text {wwiso }}+2 h_{\text {cwiso }} \cdots \cdots
\end{gathered}
$$

the related parameters are illustrated in Fig. 11.

The design map is illustrated in Fig. 12. It is a twodimensional version of the AC resistance optimum map in Fig. 10. The black line in Fig. 10 is the minimum line (3) in Fig. 12. The final minimum point is obtained along the line (1), (2), and (3). (3) is excluded if it is not in the feasible region. To obtain a final design, several design maps should be drawn to sweep all of the partial layer turns $t_{0}$.

\subsection{Winding Design with Approximation Partial} Layer Equation With the approximation equation, the winding design method is further improved. The design restrictions in (27) and (28) is modified introducing the concept of the number of layers for the partial winding $m_{p}$

$$
\begin{gathered}
d_{\mathrm{c}} \geq m_{p} d_{\mathrm{opt}}+2 m_{p} d_{\mathrm{iso}}+\left(m_{p}-1\right) d_{\mathrm{wwiso}}+2 d_{\text {cwiso }} \\
\ldots \ldots \ldots \ldots \ldots \ldots \ldots \\
h_{\mathrm{c}} \geq t d_{\mathrm{opt}}+2 t d_{\text {iso }}+(t-1) h_{\mathrm{wwiso}}+2 h_{\text {cwiso }} \cdots \cdots
\end{gathered}
$$

The design map is in Fig. 13. If there are no design restrictions, the minimum design point of each number of layers

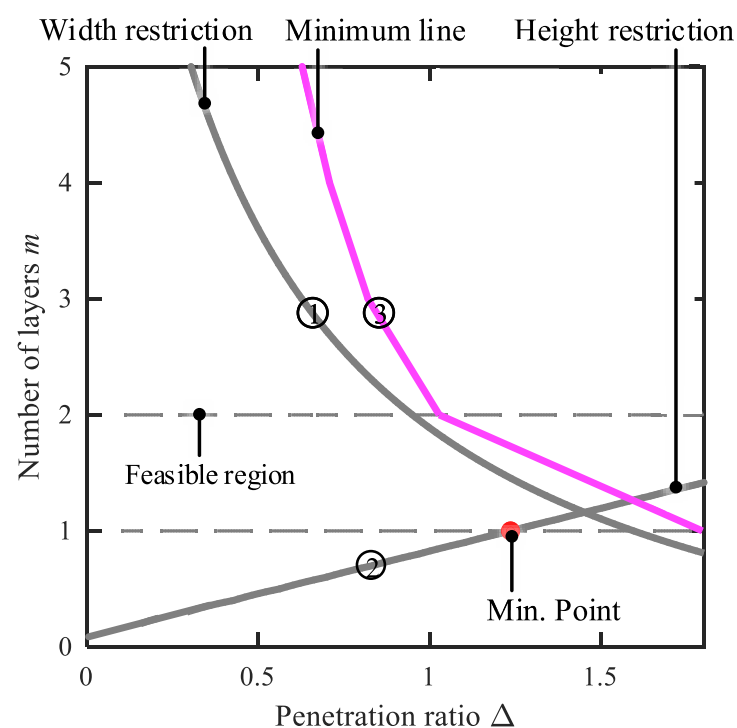

Fig. 12. Transformer winding design map with original partial layer equation

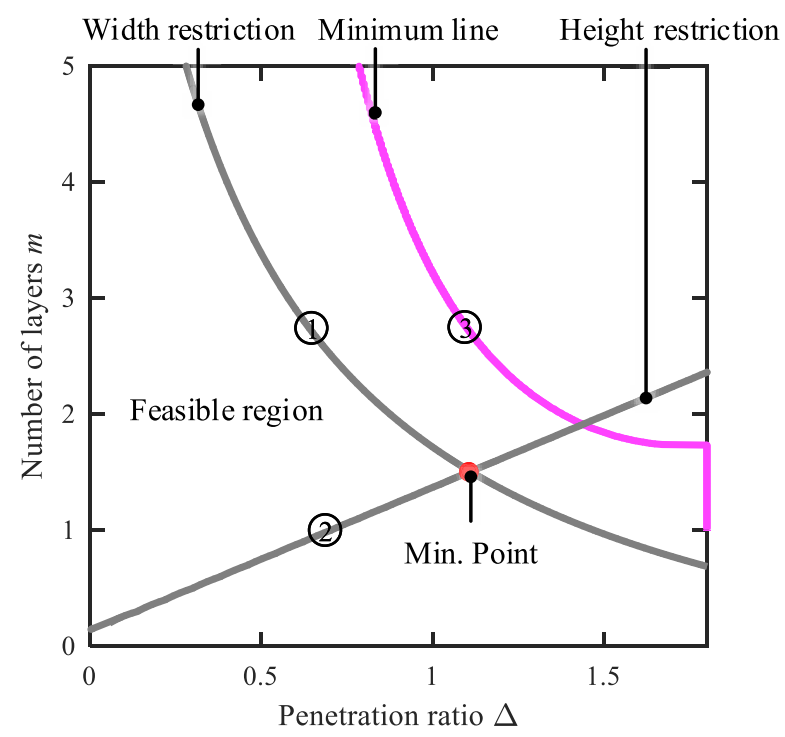

Fig. 13. Transformer winding design map with approximation partial layer equation

$m_{p}$ is located on the minimum line (3). With lines (1) and (2) defining the feasible region, the minimum point is located on those two restriction lines and line (3) in the feasible region. Once the minimum AC resistance point is obtained in the $(\Delta, m)$ plane, the wire diameter $d$ is calculated from $\Delta$. Then the winding configuration is obtained. With the improved method, only one design map is needed without sweeping all $t_{0}$ in the previous method.

The proposed method is not only applicable for sinusoidal but also for arbitrary waveform $i(t)$. The optimum thickness is obtained by changing the $z$-axis from the $\mathrm{AC}$ resistance to the total losses $P$ in Figs. 10, 12, and 13

$$
P=R_{\mathrm{dc}} I_{\mathrm{dc}}^{2}+\frac{1}{2} R_{\mathrm{dc}} \sum_{n=1}^{\infty} F_{n} I_{n}^{n} .
$$

where $I_{\mathrm{dc}}$ is the DC component of $i(t), I_{n}$ is the amplitude of the $n$th harmonic of $i(t), F_{n}$ is the AC resistance factor for the $n$th harmonic frequency. 
Table 3. Winding design input

\begin{tabular}{lcccccccc}
\hline \hline & $N$ & $p$ & $d_{\text {wwiso }}$ & $d_{\text {cwiso }}$ & $h_{\text {wwiso }}$ & $h_{\text {cwiso }}$ & $h_{\mathrm{c}}$ & $d_{\mathrm{c}}$ \\
\hline Pri. & 14 & 0.4 & $1.2 \mathrm{~mm}$ & $0.3 \mathrm{~mm}$ & $0.1 \mathrm{~mm}$ & $1.2 \mathrm{~mm}$ & $36.1 \mathrm{~mm}$ & $5.5 \mathrm{~mm}$ \\
Sec. & 34 & 0.4 & $1.2 \mathrm{~mm}$ & $0.3 \mathrm{~mm}$ & $0.1 \mathrm{~mm}$ & $1.2 \mathrm{~mm}$ & $36.1 \mathrm{~mm}$ & $5.5 \mathrm{~mm}$ \\
\hline \hline
\end{tabular}

Table 4. Winding design outputs

\begin{tabular}{lcc}
\hline \hline Design method & $\begin{array}{c}\text { Primary } \\
\text { Ori. / Approx. }\end{array}$ & $\begin{array}{c}\text { Secondary } \\
\text { Ori. / Approx. }\end{array}$ \\
\hline Number of full layers $m$ & 1 & 1 \\
Turns of full layer $t$ & 14 & 22 \\
Number of partial layers & 0 & 1 \\
Turns of partial layer $t_{0}$ & 0 & 12 \\
Wire diameter $d$ output $(\mathrm{mm})$ & $0.84 / 0.86$ & $0.65 / 0.64$ \\
Wire diameter $d$ chosen $(\mathrm{mm})$ & 1.04 & 0.62 \\
AC resistance $R_{\mathrm{ac}}$ at $20 \mathrm{kHz}(\Omega)$ & 0.0280 & 0.2094 \\
Total AC resistance & \multicolumn{3}{c}{0.0635} \\
reflected to primary $R_{\text {tot }}(\Omega)$ & \multicolumn{3}{|c}{} \\
\hline \hline
\end{tabular}

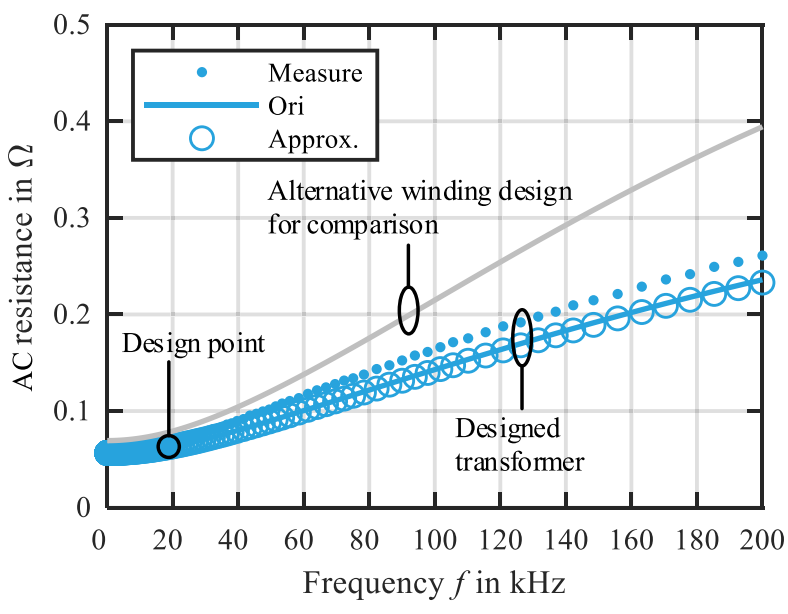

Fig. 14. Experimental result of the designed transformer with minimum winding losses

3.4 A Case Study of Optimum Winding Design To verify the design method, a case study for the winding design of a transformer in an LLC converter is presented. The transformer operates at $20 \mathrm{kHz}$. PQ50/50 and 3C91 are chosen as the core shape and material, respectively. The inputs for the winding design is in Table 3. Applying the design methods with the original partial layer and approximation equations, the design results are illustrated in Table 4. The design maps are with Figs. 12 and 13, respectively.

Firstly, for both design methods, the input only needs the number of turns $N$ and the window dimensions. The number of layers $m$ is the design output instead of input, which is of important practical value. Secondly, the original partial layer design method sweeps $t_{0}$, therefore, approximate $N / 2$ design maps are needed. In contrast, the approximation method only needs one design map with the concept of $m_{p}$. Thirdly, both design methods output the same designed $m, t, t_{0}$. There are negligible differences in the wire diameter output $d$.

The designed transformer is built and tested in Fig. 14. The primary winding is at the inner position and closer to the symmetry axis than secondary winding. The experimental, original partial layer, and approximation equation results show a good agreement in a wide frequency range. An alternative design that also fits the design restrictions is given for comparison. The core and primary winding is the same as the designed one. The secondary is with 2 full layers, 17 turns in each layer, $0.25 \mathrm{~mm}$ diameter wire. In the design point at $20 \mathrm{kHz}$, The optimum design results are with $24.85 \%$ AC resistance reduction compared with the alternative design.

\section{Conclusions}

An approximation model for the AC resistance of the partial layer is proposed for windings of inductors and transformers. With the concept of the number of layers for the partial winding, the proposed model is simpler compared with the original partial layer equation. The maximum AC resistance difference between them is $0.21 R_{\mathrm{dc}}$. The difference decreases dramatically and is negligible with the increase in the number of layers. The model is verified by the finite element method simulation and experimental results in two case studies. Finally, a design method based on the approximation equation is proposed. It is practical, which only needs the window dimensions and the number of total turns as design inputs. A case study for the winding design of a transformer in a $20 \mathrm{kHz}$ LLC converter is presented. The proposed design method results in $24.85 \%$ AC resistance reduction compared with an alternative design.

\section{References}

( 1 ) J.-P. Vandelac and P.D. Ziogas: "A Novel Approach for Minimizing Highfrequency Transformer Copper Losses", IEEE Trans. Power Electron., Vol.3, No.3, pp.266-277 (1988)

( 2 ) I. Villar: Multiphysical Characterization of Medium-frequency Power Electronic Transformers, PhD thesis, Lausanne, Switzerland: École Polytechnique Fédérale de Lausanne (2010)

( 3 ) J. Muehlethaler: Modeling and Multi-objective Optimization of Inductive Power Components, PhD thesis, Zurich, Switzerland: ETH Zurich (2012)

( 4 ) M. Nakahara and K. Wada: "Loss Analysis of Magnetic Components for a Solid-State-Transformer", IEEJ Trans. IA, Vol.4, No.4, pp.387-394 (2015)

( 5 ) P.L. Dowell: "Effects of Eddy Currents in Transformer Windings", Proc. Inst. Electr. Eng., Vol.113, No.8, pp.1387-1394 (1966)

( 6 ) J.A. Ferreira: "Improved Analytical Modeling of Conductive Losses in Magnetic Components", IEEE Trans. Power Electron., Vol.9, No.1, pp.127-131 (1994)

( 7 ) J. Lammeraner and M. Štafl: Eddy Currents, London, U.K.: Iliffe (1966)

( 8 ) J.A. Ferreira: "Appropriate Modelling of Conductive Losses in the Design of Magnetic Components", Proc. IEEE Power Electron. Spclst. Conf., pp.780785 (1990)

( 9 ) M.K. Kazimierczuk: High-frequency Magnetic Components, West Sussex, UK: John Wiley \& Sons (2009)

(10) G.S. Dimitrakakis, E.C. Tatakis, and E.J. Rikos: "A New Model for the Determination of Copper Losses in Transformer Windings with Arbitrary Conductor Distribution under High Frequency Sinusoidal Excitation", Proc. Eur. Conf. Power Electron. Appl., pp.1-10 (2007)

(11) C.R. Sullivan: "Optimal Choice for Number of Strands in a Litz-wire Transformer Winding", IEEE Trans. Power Electron., Vol.14, No.2, pp.283-291 (1999)

(12) R. Wojda and M.K. Kazimierczuk: "Winding Resistance of Litz-wire and Multi-strand Inductors", IET Power Electron., Vol.5, No.2, pp.257-268 (2012)

(13) V. Vaisanen, J. Hiltunen, J. Nerg, and P. Silventoinen: "Ac Resistance Calculation Methods and Practical Design Considerations when Using litz Wire", In Proc. IEEE 39th Annual Conf. Ind. Electron. Soc., pp.368-375 (2013)

(14) M.A. Bahmani, T. Thiringer, and H. Ortega: "An Accurate Pseudoempirical Model of Winding Loss Calculation in HF Foil and Round Conductors in Switchmode Magnetics", IEEE Trans. Power Electron., Vol.29, No.8, pp.4231-4246 (2014)

(15) D. Leuenberger and J. Biela: "Semi-Numerical Method for Calculation of Loss in Foil Windings Exposed to an Air-Gap Field", IEEJ Trans. IA, Vol.4, No.4, pp.301-309 (2015)

(16) Z. Shen, Z. Li, L. Jin, and H. Wang: "An AC Resistance Optimization Method Applicable for Inductor and Transformer Windings with Full Layers and 
Partial layers", Proc. IEEE APEC Exposit., 2017, pp.2542-2548 (2017)

(17) W.G. Hurley and W.H. Wolfle: Transformers and Inductors for Power Electronics: Theory, Design and Applications, West Sussex, UK: John Wiley \& Sons (2013)

(18) Z. Shen, H. Wang, Y. Shen, Z. Qin, and F. Blaabjerg: "An Improved Stray Capacitance Model for Inductors", IEEE Trans. Power Electron., Vol.34, No.11, pp.11153-11170 (2019)

(19) D. Meeker: Finite Element Method Magnetics (FEMM), Version 4.2 (2016)

Zhan Shen (Non-member) received the B.E. degree in electrical en-

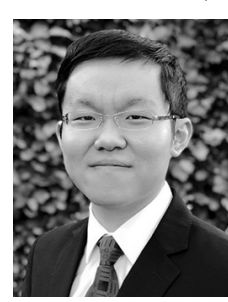
gineering and automation from Nanjing University of Aeronautics and Astronautics in 2013, and M.E. degree in electrical engineering from Southeast University in 2016, both in Nanjing, China. He is a Research Assistant at Southeast University, Nanjing, China, and the Center of Reliable Power Electronics (CORPE), Aalborg University, Aalborg, Denmark. He was a Visiting Student and pursued his master thesis at the RWTH Aachen University, Aachen, Germany, from Oct. 2014 to Feb. 2016, and a Visiting Scholar with the Massachusetts Institute of Technology (MIT), Cambridge, MA, USA, from Oct. 2018 to Jan. 2019. He was with the ABB Corporate Research Center, Beijing, China, in 2016. His research interests include the electromagneticthermal-reliability modeling and design of magnetic components in power electronic converters.

Zhiguang Li (Non-member) received the B.S. degree in electrical en-

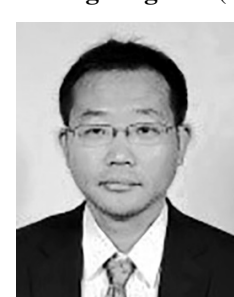
gineering from Taiyuan University of Technology, Taiyuan, China, in 1997, and M.E. degree in electrical engineering from Tianjin University, Tianjin, China, in 2000 , respectively. He is currently pursuing the Ph.D. degree at Southeast University, Nanjing, China. His current research interests include power electronics and design of medium-frequency transformer.
Long Jin (Non-member) received the master degree in automation,

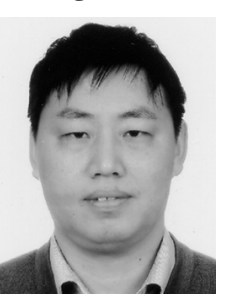
and $\mathrm{Ph} . \mathrm{D}$. degree in vibration, concussion, and noises from the Nanjing University of Aeronautics and Astronautics, Nanjing, China, in 1993 and 1997, respectively. He is currently a Professor with the School of Electrical Engineering, Southeast University, Nanjing, China. His current research interests include the design and control of ultrasonic motors, high-power electronics, and robotics.

Huai Wang (Non-member) received the B.E. degree in electrical en-

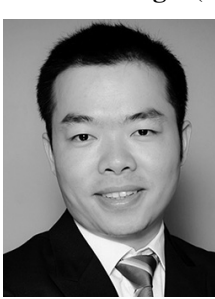
gineering, from Huazhong University of Science and Technology, Wuhan, China, in 2007 and the Ph.D. degree in power electronics, from the City University of Hong Kong, Hong Kong, in 2012. He is currently Professor with the Center of Reliable Power Electronics (CORPE), Department of Energy Technology at Aalborg University, Denmark. He was a Visiting Scientist with the ETH Zurich, Switzerland, from Aug. to Sep. 2014, and with the Massachusetts Institute of Technology (MIT), USA, from Sep. to Nov. 2013. He was with the ABB Corporate Research Center, Switzerland, in 2009. His research addresses the fundamental challenges in modelling and validation of power electronic component failure mechanisms, and application issues in system-level predictability, condition monitoring, circuit architecture, and robustness design. Dr. Wang received the Richard M. Bass Outstanding Young Power Electronics Engineer Award from the IEEE Power Electronics Society in 2016, and the Green Talents Award from the German Federal Ministry of Education and Research in 2014. He is currently the Chair of IEEE PELS/IAS/IES Chapter in Denmark. He serves as an Associate Editor of IET Electronics Letters, IEEE JOURNAL OF EMERGING AND SELECTED TOPICS IN POWER ELECTRONICS, and IEEE TRANSACTIONS ON POWER ELECTRONICS. 